\title{
Experimental method for the evaluation of the susceptibility of materials to shear band formation
}

\author{
R. Tham \\ Fraunhofer Institute for High-Speed Dynamics, Ernst-Mach-Institut, EMI, 79104 Freiburg, Germany
}

\begin{abstract}
In order to characterize materials with respect to their susceptibility to shear band formation at high strain rates, a modified Hopkinson pressure bar apparatus and hat-shaped steel specimens with a shear zone having a width significantly larger than the typical width of adiabatic bands are used. The sample is directly impacted by the striker. The force acting on the sample is measured with a PVDF-gauge between the sample and the output bar. The displacement is recorded with an electrooptical extensometer. The energy absorbed by the shearing process up to failure can be used as a reference for the susceptibility of materials to shear band formation. The method is demonstrated comparing the shear behavior of two high-strength steels with similar metallic structure and strength. Differences were found in the transition region between quasi-static and fully adiabatic shearing conditions where the energy up to rupture differs by $40 \%$. For fully adiabatic shear band formation, the deformation process of both materials equals. At extreme rates, shear processes are mainly governed by the thermodynamic properties of the materials. On the other hand, strength and structural properties play a role for low and intermediate rates where global and localized shear mechanisms occur in parallel.
\end{abstract}

\section{Introduction}

In many industrial applications, materials are subjected to fast shear loading and fail by localized shear associated with the occurrence of adiabatic shear bands (ASB). These processes are mainly governed by the thermo-mechanical properties of the materials. A comprehensive treatise on the phenomenon of adiabatic shearing is the work of Bai and Dodd [1]. Only little attention was paid to the transition region between quasi-static shear processes and pure adiabatic shear. For shear rates allowing both processes, strength properties as well as thermo-mechanical characteristics govern failure. In order to establish criteria for the susceptibility of materials, experiments are performed using a specimen geometry allowing non-localized shear and adiabatic shear, as well. Materials of comparable structure are investigated, strength parameters differ little. The loading velocity is in the range between quasi-static values and more than $15 \mathrm{~m} / \mathrm{s}$ to reach extreme shear rates.

\section{Experimental approaches}

The materials used for the comparative study were two low-carbon martensitic steels, denoted M1 and M2, with slightly different strength and elongation at fracture. Tensile properties are given in table 1 , where $\mathrm{C}$ is the carbon content, $R_{p, 0.2}$ the yield strength at $0.2 \%$ elongation, $R_{m}$ the UTS and $\mathrm{A}_{5}$ the elongation at fracture.

In order not to force adiabatic shearing by a superimposed pressure component, not the hydrodynamic specimen geometry (Couque [2]) but the conventional hat-shaped specimen was preferred. The dimensions of the samples together with a view of the machined specimen are shown in figure 1 . The nominal length of the shear zone is $1 \mathrm{~mm}$, its width $0.3 \mathrm{~mm}$. These values were measured for each individual component after fabrication. Thus, the width is much larger than the typical width of adiabatic bands and non-localized shear is enabled. Shear is initiated by compression of the sample; the hat has a cap in order to reduce the stress at the interfaces between the loading device and the specimen.

In quasi-static experiments $(0.02 \mathrm{~mm} / \mathrm{s})$, the load was applied to the specimens by means of a servo-hydraulic material testing device with polished compression platens. The force acting upon the sample was measured with an accuracy of $0.5 \%$. The same precision was achieved for the displacement measurement by means of an extensometer clipped to the compression platens.

Dynamic experiments were carried out using a modified Hopkinson pressure bar apparatus with direct impact of the striker on the specimen, which is in contact with the output bar. The impact velocities ranged between $4.7 \mathrm{~m} / \mathrm{s}$ and $15.3 \mathrm{~m} / \mathrm{s}$, thus resulting in nominal shear strain rates between $1.6 \times 10^{4} 1 / \mathrm{s}$ and $5.1 \times 10^{4} 1 / \mathrm{s}$. As a result of decreasing velocity of the striker, lower rates can be observed, especially in the low velocity regime. Both output bar and striker had a diameter of $22 \mathrm{~mm}$, the length of the first was $2200 \mathrm{~mm}$, that of the striker $300 \mathrm{~mm}$. The pressure bar is made of high-strength aluminum, the impacting bar, depending on velocity and energy consumption of the specimen, of $\mathrm{Al}$, steel or a tungsten alloy with a density of $17.5 \mathrm{~g} / \mathrm{cm}^{3}$. More details of the set-up can be seen in figure 2. Besides the gas accelerator (1), the striker (2) and the output bar (3), the measurement instrumentation is displayed: In contact with the sample is the PVDFload sensor (4), the impact velocity is measured by means of laser-light barrier (5), the shear displacement of the sample is recorded by an electro-optical extensometer (6) at a resolution of $20 \mu \mathrm{m}$ and with a limiting frequency of $250 \mathrm{kHz}$.

An explicit view of the sample area together with the load sensor is given in figure 3 . The shoulders of the specimen's groove are followed by the extensometer. Thus, the relative displacement of the two shear surfaces can be measured yielding the shear strain. The PVDF-sensor 
Table 1. Tensile properties of the steels under study.

\begin{tabular}{|l|c|c|c|c|}
\hline & $\mathrm{C} \%$ & $\mathrm{R}_{\mathrm{p}, 0.2} \mathrm{MPa}$ & $\mathrm{R}_{\mathrm{m}} \mathrm{MPa}$ & $\mathrm{A}_{5} \%$ \\
\hline Mat1 & 0.16 & 997 & 1018 & 16 \\
\hline Mat2 & 0.32 & 1020 & 1087 & 14 \\
\hline
\end{tabular}
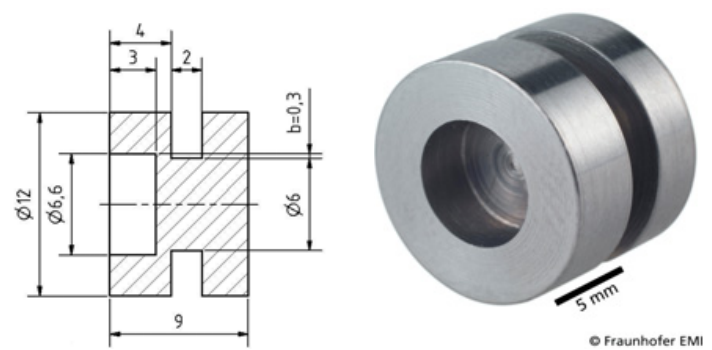

Fig. 1. Geometry of the specimens.

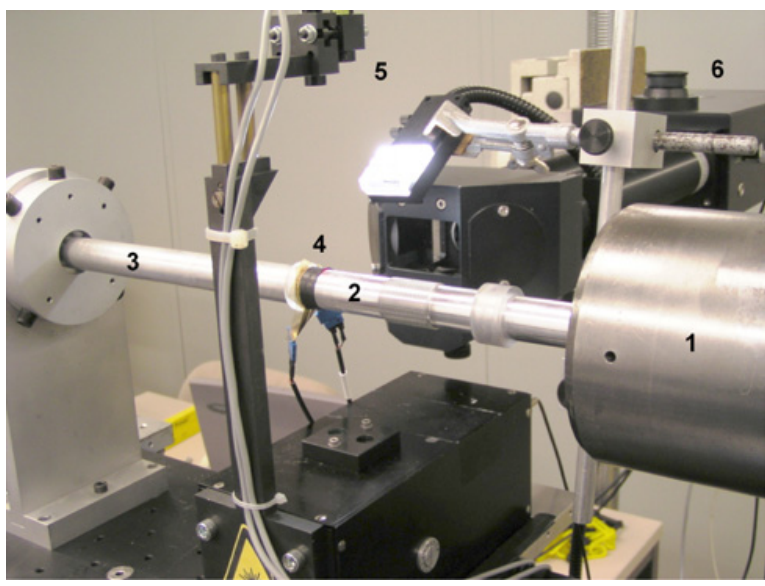

Fig. 2. Hopkinson bar and measuring devices.

between the sample and the output bar has the same active area as the bars. It is encapsulated between two ultra-hard steel platens of $2 \mathrm{~mm}$ thickness, centered to the output bar by means of a plastic ring and coupled to it with highly viscous grease. Additionally, the force can be measured on the output bar by strain gauges in a Wheatstone bridge circuit. Hereby, the PVDF-gauge is calibrated once. A nonlinear calibration function was found yielding a nearly constant gauge sensitivity of $10.3 \mathrm{pC} / \mathrm{N}$ for forces exceeding $12 \mathrm{kN}$, and approaching a value of $11.3 \mathrm{pC} / \mathrm{N}$ for forces close to 0 . The direct measurement of the force acting upon the sample is favored due to much less superimposed oscillations compared to the strain gauge technique, especially at the beginning of loading. The use of the gauge is limited to a loading time below $4 \mu$ s beyond which viscoelastic deformation of the PVDF-foil influences its sensitivity. More information on the physics and use of PVDF-gauges can be found in $[3,4]$.

\section{Data evaluation}

Data were recorded at a sample rate of $1 \mathrm{MHz}$. With the directly measured time-dependent force $\mathrm{F}(\mathrm{t})$ acting parallel to the shear surface of the specimen, the shear stress is calculated by dividing force by the inner ring surface

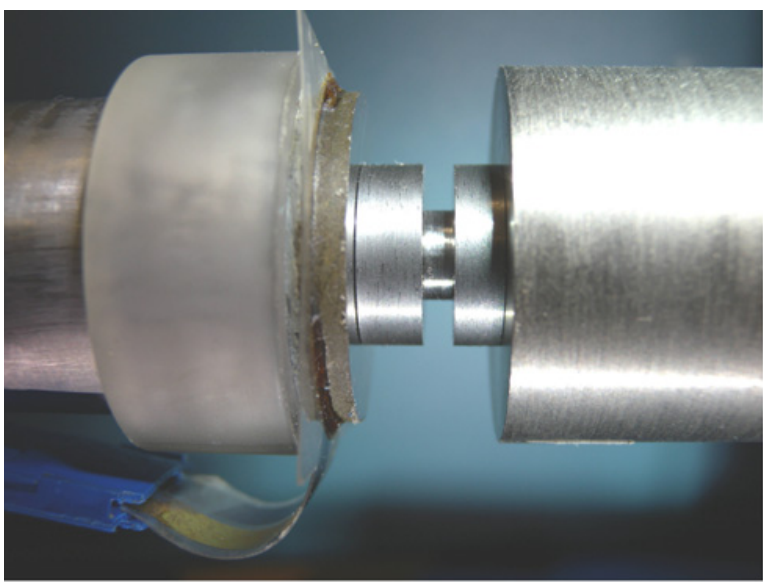

Fig. 3. Detailed view of the sample area of the Hopkinson bar.

$\left(18.85 \mathrm{~mm}^{2}\right)$ of the shear volume. Here, the maximum shear stress is apparent. Due to the small displacements $\mathrm{x}(\mathrm{t})$ recorded, these data were smoothed by fitting them with a high-order polynomial regression function in order to reduce jitter and allowing mathematical differentiation. Conventionally, the shear strain is deduced by dividing the displacement of the inner ring surface by the width of the shear zone $(0.3 \mathrm{~mm})$. Differentiation of the time-dependent shear strain curve results in a strain rate at each moment of loading. For stress values $\geq 550 \mathrm{MPa}$ (assuming plastic behavior), the mean is taken as the average strain rate of the deformation process.

A measure of the materials' susceptibility to shear band failure is assumed to be the total plastic work up to fracture. This value is calculated by a numerical integration of the smoothed curve $\mathrm{F}(\mathrm{x})$.

\section{Results}

In the following, the shear behavior of the two materials as result of the average strain rate is displayed by stress-strain diagrams. Micrographs of the deformed structure reveal the mechanisms.

The influence of strain rate on the stress-strain curve can be seen in figure 4 . It has to be pointed out that the mentioned rates are considered as global ones, in the case of deformation localization and the formation of ASB, much higher values are reality and are accessible by measuring the width of the shear zone by microscopic inspection. Compared to the quasi-static test, the maximum stress is slightly increased in the dynamic regime, the shear strain at fracture reduces drastically with increasing rate. Due to thermal softening associated with fast loading, the stress level reduces already for low shear values. At the highest rate, the localization of shear in a narrow adiabatic band is associated with a steep drop of the shear stress at a strain of 0.3 . The area below the curves is a measure for the plastic work. It is obvious that with increasing rate less energy is consumed.

In the static test, the deformation is nearly homogeneously distributed over the width of the shear zone and rupture occurs at the inner surface of the ring-shaped shear 


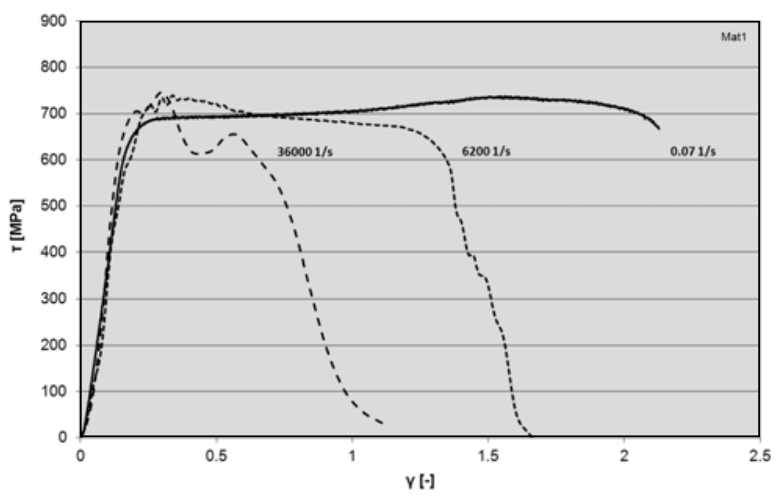

Fig. 4. Stress-strain diagram of Mat1 at three strain rates.

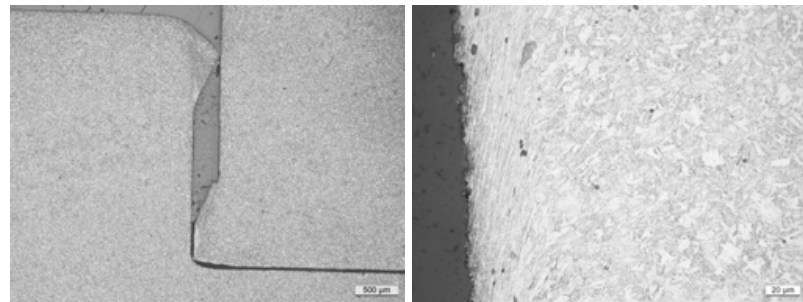

Fig. 5. Failure und microstructure of Mat1 loaded at 6200 1/s.

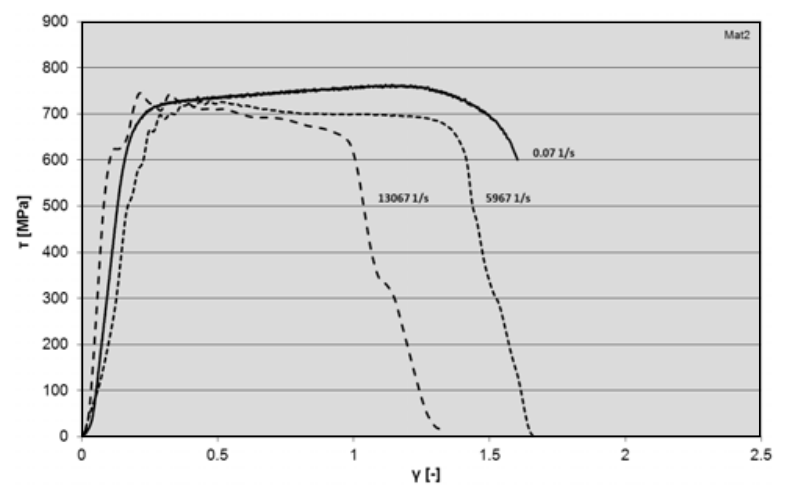

Fig. 6. Stress-strain diagram of Mat2 at three strain rates.

volume. At a rate of $62001 / \mathrm{s}$, shear localizes to both sides of the crack in a width of approximately $35 \mu \mathrm{m}$, see figure 5. The rupture is diagonal from the inner to the outer edge of the ring's cross section. With still increasing strain rate, the zone of large shear strain is getting narrower, phase transitions (white lines) were not observed. Due to the total rupture of the specimen, such effects are not accessible to a microscopic inspection.

The relation between stress and strain of Mat2 for various strain rates is depicted in figure 6 . The highest rate of Mat1 was not reached in these tests, therefore, pure adiabatic shear leading to failure was not observed. Under quasi-static conditions, Mat2 shows a slightly increased stress level which is due to the higher strength of the material. The maximum shear at fracture is reduced. In the dynamic regime, the behavior is comparable. Microstructural mechanisms correspond to those of Mat1.

The aforementioned results do not clearly yield the criteria for the susceptibility of materials to adiabatic shear failure. An attempt to find a more distinct measure was made in calculating the energy of the shear process up to

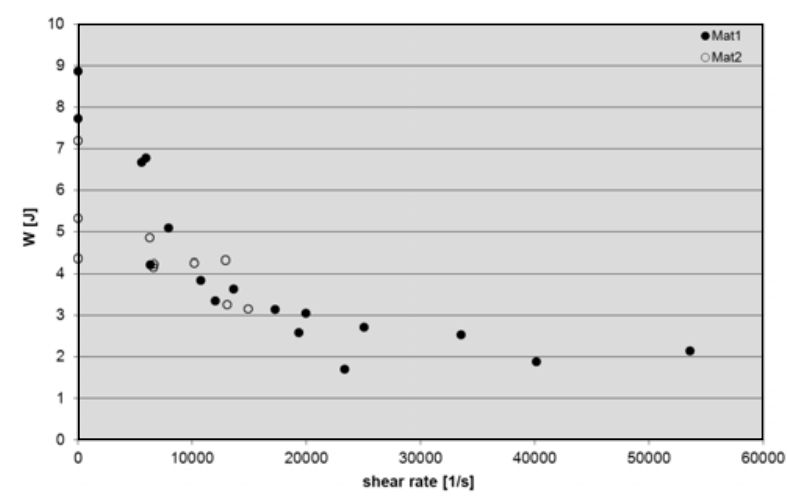

Fig. 7. Work up to rupture.

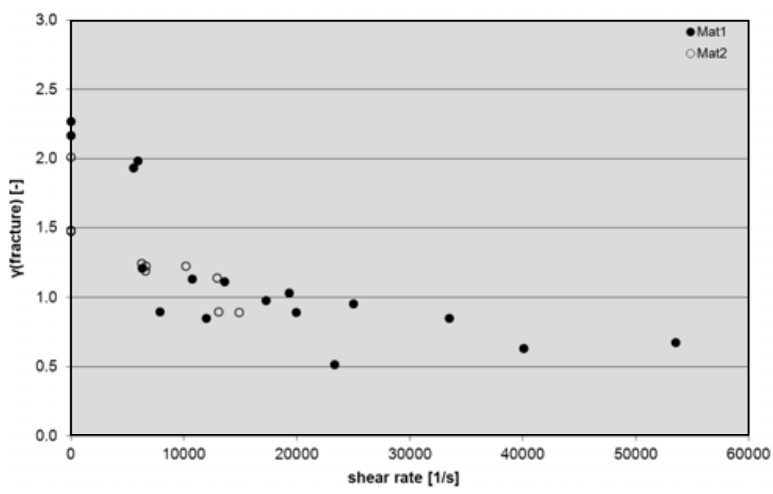

Fig. 8. Relation between plastic strain at fracture and rate.

rupture and in correlating these values with shear rate, see figure 7.

Differences in the materials' behavior are obvious for shear rates below $10^{4} 1 / \mathrm{s}$. The more ductile material Mat1 allows a larger deformation over the whole width of the specimen before the onset of localized shear and, therefore, more deformation energy is consumed. Because of shear localization these, differences disappear at higher rates. Experiments at rates exceeding $1.5 \times 10^{4} 1 / \mathrm{s}$ were only carried out for Mat1 because of the fact that here both materials behave in a comparable manner. Above $2.5 \times$ $10^{4} 1 / \mathrm{s}$, a total localization of deformation in narrow bands of a few tenths of micrometers is apparent. The work done up to fracture is constant reaching a value of approximately $2 \mathrm{~J}$. In tests using a specimen having a reduced width of the shear zone of $0.1 \mathrm{~mm}$, the same tendency was observed, except the fact that differences between materials are less evident. This reveals that for smaller shear width local adiabatic deformation is comparable whereas global strain is reduced.

In order to separate stress and strain effects, the plastic strain at fracture as a function of the average strain rate is depicted in figure 8. In accordance with the aforementioned results, concerning the plastic work, rate effects upon strain are obvious for shear rates inferior to $10^{4} 1 / \mathrm{s}$. Samples of Mat1 yield higher global strain levels than those of Mat2, thus leading to larger plastic work consumed. Stress effects are of minor order. When adiabatic processes are dominant $\left(>10^{4} 1 / \mathrm{s}\right)$, the strain is concentrated in a narrow zone of much smaller size than the specimen's width and being similar for both materials. 


\section{Conclusions}

Adiabatic shear is considered to be a process taking place over a broad range of temperatures and strain rates. A method was developed to establish criteria for the tendency of materials to form adiabatic bands. The work done up to fracture is considered to be a measure of the susceptibility of materials to adiabatic shear when loaded dynamically. The experiments have shown that for comparable alloys differences in the susceptibility to ASB only play a role in a strain rate regime $<10^{4} 1 / \mathrm{s}$ where the mechanical properties compete with thermo-mechanical ones. Mechanisms at highest rates are mainly governed by thermal properties of the materials.

It has to be emphasized that in the case of shear localization only a part of the sample's cross section undergoes deformation. The mentioned shear rates are average values over the total cross section. Therefore, rates in ASB are by factors greater than the given numbers.

If structural components of large dimensions are shear loaded dynamically, the regime where non-localized deformation and the onset of ASB-formation occur in parallel is of importance for the susceptibility to later failure by pure adiabatic shear. Therefore, the focus should not be directed to extreme rates but to the intermediate range at rates between $5001 / \mathrm{s}$ and $10^{4} 1 / \mathrm{s}$. In using the same type of specimens, impact velocities between
$0.15 \mathrm{~m} / \mathrm{s}$ and $3 \mathrm{~m} / \mathrm{s}$ will result. The Hopkinson pressure bar is not suitable for this kind of experiments because of the lack of energy at low velocities. High-speed servohydraulic material testing systems meet the requirements. The PVDF-load measurement technique used in the preceding experiments is already tested in this application and found to be preferable to the standard quartz gauges.

In order to investigate the on-going micro-structural processes by means of microscopic inspection, fracture should be avoided. This will be done by a slight modification of the specimen's geometry only allowing a limited shear.

\section{References}

1. Y. Bai, B. Dodd, Adiabatic Shear Localization, (Pergamon Press, Oxford, 1992)

2. H. Couque, J. Phys. IV France 110 (2003), (EDP Sciences, Les Ulis)

3. F. Bauer, H. Moulard, Réponse sous choc de polymères ferroélectriques: Capteur piézoélectrique PVDF nanosecond, (ISL-Rapport PU 381/95, SaintLouis, 1995)

4. F. Bauer, Mat. Res. Soc. Symp. Proc. Vol. 698 (2002), (Materials Research Society) 